\title{
Serum miRNAs in women affected by hyperandrogenic polycystic ovary syndrome: the potential role of miR-155 as a biomarker for monitoring the estroprogestinic treatment
}

\author{
Walter Arancio, Marco Calogero Amato, Miriam Magliozzo, Giuseppe \\ Pizzolanti, Rosalia Vesco \& Carla Giordano
}

To cite this article: Walter Arancio, Marco Calogero Amato, Miriam Magliozzo, Giuseppe Pizzolanti, Rosalia Vesco \& Carla Giordano (2018): Serum miRNAs in women affected by hyperandrogenic polycystic ovary syndrome: the potential role of miR-155 as a biomarker for monitoring the estroprogestinic treatment, Gynecological Endocrinology, DOI: 10.1080/09513590.2018.1428299

To link to this article: https://doi.org/10.1080/09513590.2018.1428299

$+\quad$ View supplementary material $\longleftarrow$

册 Published online: 31 Jan 2018.

Submit your article to this journal $₫$

View related articles $\sqsubset$

View Crossmark data $₫$ 


\title{
Serum miRNAs in women affected by hyperandrogenic polycystic ovary syndrome: the potential role of miR-155 as a biomarker for monitoring the estroprogestinic treatment
}

\author{
Walter Arancio* (D), Marco Calogero Amato*, Miriam Magliozzo, Giuseppe Pizzolanti, Rosalia Vesco and \\ Carla Giordano
}

Section of Endocrinology, Diabetes and Metabolism, Biomedical Department of Internal and Specialized Medicine (Di.Bi.M.I.S), University of Palermo, Palermo, Italy

\begin{abstract}
MicroRNAs can be used as very efficient circulating biomarkers. The role of microRNAs in polycystic ovary syndrome (PCOS) and the effects of antiandrogen therapy on microRNA expression is still not fully understood. A panel of serum microRNAs were retrotranscribed via looped reverse primer transcription specific for each miRNA and quantified via probe specific RT-PCR in 16 Caucasian hyperandrogenic PCOS women selected according to the Rotterdam criteria and in a subset of seven patients after four months of sequential reverse antiandrogenic therapy. All women recruited underwent an oral glucose tolerance test (OGTT) and a baseline total cholesterol, high density lipoproteins cholesterol, triglycerides, AST and ALT dosage. In the follicular phase women were evaluated for total testosterone, $\Delta 4$-androstenedione, DHEAS,

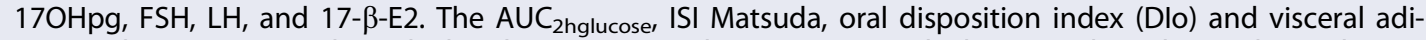
pose index (VAI) were also calculated. We suggest that miR-155 might have a role as biomarker in hyperandrogenic PCOS patients to monitor the effect of antiandrogen therapy.
\end{abstract}

\section{ARTICLE HISTORY}

Received 17 July 2017

Revised 10 January 2018

Accepted 11 January 2018

Published online 30 January

2018

\section{KEYWORDS}

Polycystic ovary syndrome; mir-155; visceral adipose index; antiandrogenic therapy; biomarker

\section{Introduction}

Polycystic Ovary Syndrome (PCOS) is the most common endocrine disorder in women, with prevalence between $6 \%$ and $10 \%$ based on the NIH criteria and about $15 \%$ when the Rotterdam criteria are applied [1]. PCOS is a complex endocrinopathy with poorly understood etiology [2] characterized by variable features, which may include: biochemical and clinical hyperandrogenism, menstrual and ovulatory dysfunctions and polycystic ovaries with exclusion of other androgenic, pituitary or adrenal causes [2]. A number of metabolic syndrome features have been associated with PCOS, such as insulin resistance, hyperinsulinemia, type 2 diabetes, dyslipidemia and cardiovascular disease and increasing lifetime risk of hypertension [3-7].

Although its etiology is still unclear, the pathogenesis of PCOS is thought to be caused by a combination of genetic, epigenetic and environmental factors [8-10]. Many studies have suggested a role for microRNAs in its pathophysiology $[8,11]$. MicroRNAs (or miRNAs) are endogenous, noncoding, small single-strand RNAs that play important regulatory roles [12]. Today miRNAs arouse strong interest in the diagnosis, treatment and prognosis of many disorders, and their role has been investigated in the pathogenesis of cancer [13], diabetes mellitus [14], cardiovascular disease [15], insulin resistance [16], endometriosis [17], inflammatory conditions [18] and many others.

Studies have investigated the role of miRNAs in the pathophysiology of PCOS using blood [19], serum [20], ovaries [21-23] and follicular fluid [23] in the regulation of the ovarian cycle [23], abnormal ovary function and follicular development
[21-22], pathogenesis of infertility [24], insulin resistance and hyperinsulinemia $[21,25,26]$. Indeed, microRNAs can be used as efficient biomarkers distinguishing PCOS from normally menstruating women and to identify specific sub-phenotypes [27].

The aim of this study is to correlate a profile of selected serum miRNAs in women with hyperandrogenic PCOS with endocrinemetabolic parameters and to assess the changes in serum miRNA after reverse antiandrogenic therapy in order to find potential Biomarker for monitoring the Estroprogestinic Treatment.

\section{Materials and methods}

\section{Patient selection}

The study was conducted on sixteen Caucasian women of reproductive age who suffered from PCOS (hyperandrogenic phenotype), selected according to the Rotterdam criteria [28]. They are part of a control group of a previous study [29]. The size of the group is suboptimal; therefore, the results must be tested in a larger population to gain major statistical significance.

All women had normal liver, kidney and thyroid functions, were not on any antihypertensive or lipid-lowering drugs, and had no other chronic pathologies reported. Other causes of hyperandrogenism were carefully excluded.

Family history of diabetes mellitus and cardiovascular disease, birth weight, age of menarche, menstrual cycle characteristics, history of hirsutism and acne and cigarette smoking were recorded. Weight, height, BMI (body mass index), waist

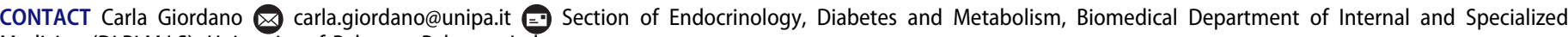
Medicine (Di.Bi.M.I.S), University of Palermo, Palermo, Italy

(4) Supplemental data for this article can be accessed here.

*The authors consider that the first two authors should be regarded as joint First Authors.

(C) 2018 Informa UK Limited, trading as Taylor \& Francis Group 
circumference, blood pressure, presence of acanthosis nigricans and striae rubrae, and hair distribution were evaluated. The presence of hirsutism was classified on the basis of a Ferriman and Gallwey score $>8$ [30].

All women recruited, underwent an oral glucose tolerance test (OGTT) and baseline total cholesterol, high density lipoproteins (HDL) cholesterol, triglycerides, aspartate transaminase (AST) and alanine transaminase (ALT) evaluation. In the follicular phase of the menstrual cycle women underwent evaluation of total testosterone, $\Delta 4$-androstenedione, dehydroepiandrosterone sulfate (DHEAS), 17-hydroxyprogesterone (17OHpg), follicle-stimulating hormone (FSH), luteinizing hormone (LH) and 17-betha-estradiol (17- $\beta$-E2). OGTT was performed after a 12-h overnight fast. The blood glucose and insulin levels, both after fasting and during the OGTT, were used to assess beta cell function $\left(\mathrm{AUC}_{2 \mathrm{~h}}\right.$ Insulin $) . \mathrm{AUC}_{2 \text { hglucose, ISI Matsuda, oral }}$ disposition index (DIo) and visceral adipose index (VAI) were also evaluated. $\mathrm{AUC}_{2 \mathrm{~h}}$ Insulin was calculated by the trapezoidal method. The DIo, that provides a measure of beta cell function [31] was calculated using the formula: ( $\Delta$ Insulina $0-30 / \Delta$ Glucose $0-30) \times(1 /$ fasting insulin) (mmol-1). ISI Matsuda, an index of insulin sensitivity, was calculated as previously reported [32,33]. The VAI, a surrogate marker of the distribution and function of adipose tissue was calculated using the formula reported in Amato et al. [6]. A subgroup of seven patients was subjected to a period of four months to sequential reverse antiandrogenic therapy with ethinyl-estradiol and cyproterone-acetate as follows: $0.01 \mathrm{mg}$ ethinyl-estradiol from 1 st to 10 th day; $0.02 \mathrm{mg}$ ethinyl-estradiol from 11 th to $21 \mathrm{st}$ day; $12.5 \mathrm{mg}$ cyproterone acetate from 1 st to 10 th day. In this group, the expression of all miRNAs was evaluated after four months of treatment.

\section{Assay methods}

The evaluations were performed at the Corelab of the Palermo university hospital. FSH, LH, 17- $\beta$-E2, 17-OH-Pg, PG, total testosterone, $\Delta 4$-androstenedione and insulin were assayed using electrochemiluminescence immunoassay ECLIA (Elecsys, Roche, Italy). Chemiluminescent assays were used for DHEAS (Immulite, Diagnostic Products, Genoa, Italy). Blood glucose, total cholesterol, HDL-cholesterol and triglycerides, ALT, AST were assayed using spectophometric methods (Modular P8000, Roche, Italy). LDL cholesterol was calculated using the Friedewald formula.

\section{RNA isolation and preparation}

Total RNA was extracted from serum samples using the miRNeasy Mini-kit (Qiagen, Hilden, Germany). The concentration and purity of RNA were estimated using a spectrophotometer NanoDrop ND-1000 (NanoDrop, Wilmington, DE, USA). The stem-loop cDNAs were synthesized using looped-reverseprimer-transcription specific for each miRNA (see below). Total RNA (10 ng) was reverse transcribed using a TaqMan ${ }^{\circledR}$ MicroRNA Reverse Transcription Kit (Applied Biosystems, Foster City, CA, USA).

\section{Analysis of miRNA gene expression}

RNU44 was used as normalizer. The expression of mature miRNAs in sera from each patient was determined by
miRNA-specific real-time PCR (RT-PCR) using a RotorGene Q-PCR system (Qiagen, Hilden, Germany). The reaction was conducted in a final volume of $20 \mu \mathrm{l}$, using TaqMan Universal PCR Master Mix (Applied Biosystems, Foster City, CA, USA). The TaqMan ${ }^{\circledR}$ primer/probe sets used are the following: RNU44 (001094), hsa-miR-9 (000583), hsa-miR-21 (000397), hsa-miR27b (000409), hsa-miR-29a (002112), hsa-miR-30a-3p (000 416), hsa-miR-103 (000439), hsa-miR-124a (000446), hsa-miR-155 (002623), hsa-miR-223 (002295), hsa-miR-320 (002277), hsa-miR -375 (000564), (Applied Biosystems, Foster City, CA, USA). The relative quantification of the expression was performed using the Ct method (2- $2^{-\mathrm{Ct}}$ method) [34].

The miRNAs to analyze have been selected from literature because reported to be related to PCOS and associated phenotype (please refer for the details to the review of Ilie and Georgescu in 11 , but also to $12-31$ ).

\section{Statistical analyses}

The statistical analyzes were performed with SPSS software v. 17 for Windows. Quantitative variables were expressed as the mean and standard deviation, and categorical variables as the number and percentage frequency. The differences between the unpaired quantitative data were calculated with the Mann-Whitney $U$-test. The correlations between quantitative variables were assessed using the Spearman Rho test. The differences between paired quantitative data (before and after four months of therapy) were evaluated with the Wilcoxon test. Values of $p<0.05$ were considered significant.

\section{Ethical approval}

Women recruited for the study were followed at our endocrinological gynecology clinic in Palermo from 2013 to 2014. All procedures performed in studies involving human participants were in accordance with the Helsinki declaration. Informed consent was obtained from all participants included in the study. The participants remained anonymous during data analyses. The ethical committee "Palermo1" of the university hospital "Paolo Giaccone" approved the procedure (11/2015).

\section{Results}

The clinical, anthropometric and hormonal characteristics of patients are shown in (S1). The expression levels of miRNAs at the beginning of the study are reported in (S2).

Women with oligo-amenorrhea compared to eumenorrheic women exhibited significantly higher expression of miR-30a-3p $(p=0.009)$ and miR-124a (S3-S4). No difference was found regarding the expression of miRNAs in relation to the presence of polycystic ovarian morphology (S3).

A univariate analysis was performed to identify possible correlations between miRNAs and hormonal profile; androstenedione presented significant inverse association with miR-155 (Rho $=-0.505, \quad p=0.046) \quad$ and miR-29a $\quad(\mathrm{Rho}=-0.541$, $p=0.030)$, the 17-OHPg with miR-155 $(\mathrm{Rho}=-0.666, p=0.005)$ (Figure 1(A-C); S5). Concerning the metabolic parameters, DIo is significantly associated with $\mathrm{miR}-103$ (Rho $=0.612, p=0.012$ ), and LDL cholesterol was inversely correlated with miR-30a-3p $(\mathrm{Rho}=-0.593, p=0.016)$ and $\operatorname{miR}-320(\mathrm{Rho}=-0.512, p=0.043)$ (Figure 1(D,F); S6). 

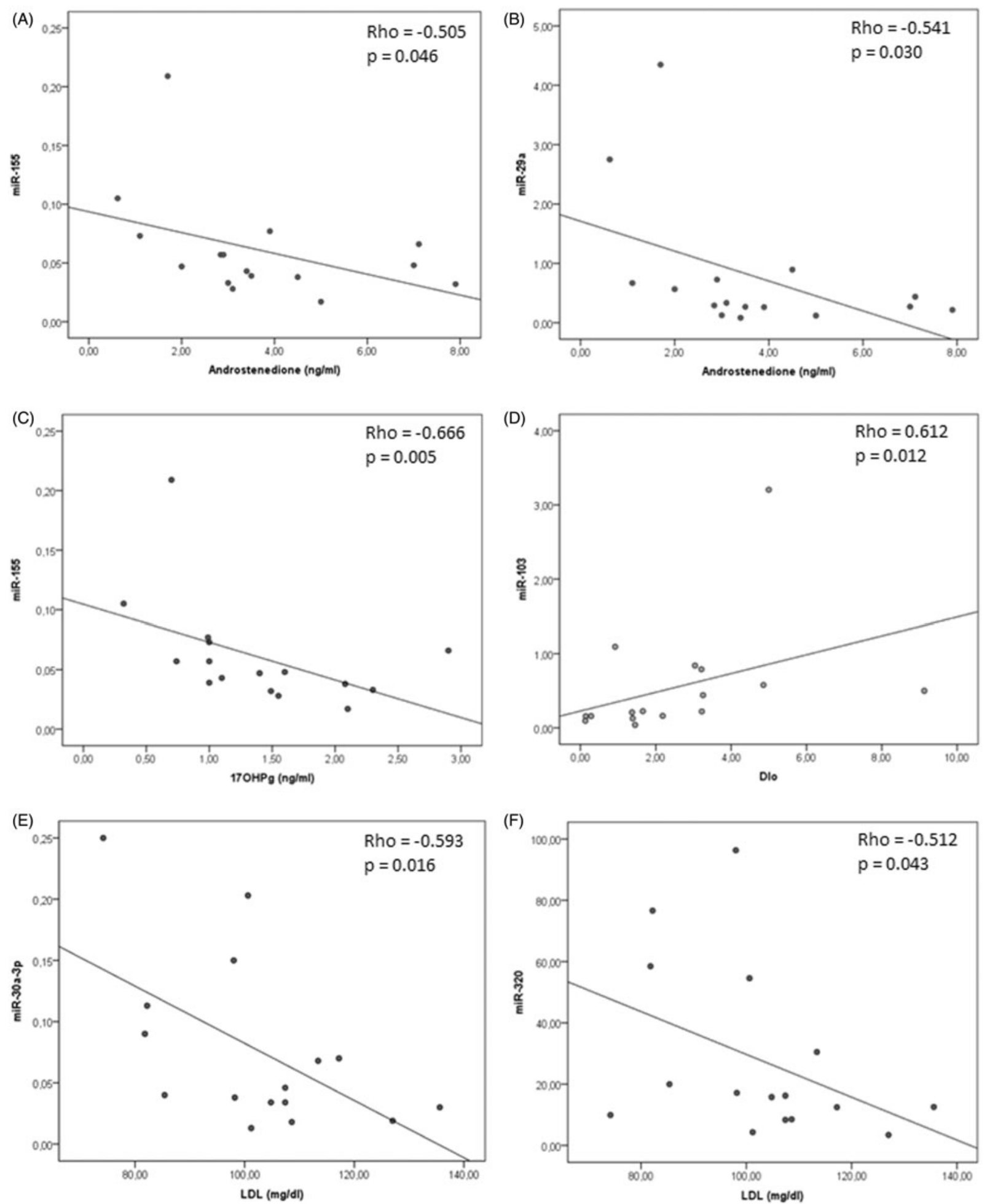

Figure 1. Univariate analysis of significant correlations between miRNAs and hormonal and metabolic parameters. Univariate analysis points to a correlation between expression of miR-155 and expression of Androstenedione (A), expression of miR-29a and expression of Androstenedione (B), expression of miR-155 and expression of $170 \mathrm{HPg}(\mathrm{C})$, expression of miR-103 and Dlo (D), expression of miR-30a-3p and LDL quantification (E), and expression of miR-330 and quantification of LDL (F) in PCOS patients.

We also evaluated the effect of antiandrogen therapy on miRNA expression in a subset of seven patients undergoing sequential reverse antiandrogenic therapy of Hammerstein [35] for a period of four months: the therapy induced a significant increase in the expression of $\mathrm{miR}-27 \mathrm{~b} \quad(0.044 \pm 0.016$ vs. $0.201 \pm 0.156 ; p=0.043)$ and miR-155 $(0.05 \pm 0.02$ vs. $0.11 \pm 0.70$; $p=0.034)$ (Table 1; S7).

\section{Discussion}

The present study aimed to characterize a profile of serum miRNAs in women with PCOS (hyperandrogenic phenotype) and to evaluate possible correlations with endocrine-metabolic parameters. The serum expression of 11 miRNA selected from literature as potentially involved in the phenotype under study 
Table 1. Anti-androgen therapy effects on the miRNAs expression.

\begin{tabular}{lccc}
\hline & Basal & After four months of therapy & \\
& Mean \pm SD & Mean \pm SD & $p$ \\
\hline miR-27b & $0.044 \pm 0.016$ & $0.201 \pm 0.156$ & 0.043 \\
miR-223 & $23.05 \pm 43.23$ & $16.20 \pm 18.48$ & 1 \\
miR-155 & $0.05 \pm 0.02$ & $0.11 \pm 0.7$ & 0.034 \\
miR-375 & $0.19 \pm 0.09$ & $0.28 \pm 0.26$ & 1 \\
miR-29a & $0.74 \pm 0.92$ & $1.13 \pm 0.99$ & 0.398 \\
miR-30a-3p & $0.06 \pm 0.04$ & $0.08 \pm 0.07$ & 0.799 \\
miR-21 & $2.43 \pm 1.92$ & $4.30 \pm 3.13$ & 0.063 \\
miR-9 & $0.003 \pm 0.001$ & $0.006 \pm 0.005$ & 0.279 \\
miR-103 & $0.70 \pm 1.11$ & $1.56 \pm 1.90$ & 0.398 \\
miR-124a & $0.001 \pm 0.001$ & $0.002 \pm 0.002$ & 0.207 \\
miR-320 & $32.52 \pm 32.74$ & $50.02 \pm 66.32$ & 0.463 \\
\hline
\end{tabular}

The expression of miRNAs and the statistical significance of their differential expression comparing patients before and after the anti-androgen therapy is reported. In bold the statistically significant difference at $p<0.05$ is reported.

[11-31], namely miR-9, miR-21, miR-27 b, miR-29a, miR-30a-3p, miR-103, miR-124a, miR-155, miR-223, miR-320, miR-375, was evaluated in 16 Caucasian women of reproductive age who suffered from PCOS (hyperandrogenic phenotype). The changes in expression of miRNAs, caused by a four-month antiandrogen therapy were evaluated in a subset of seven women.

The comparative analysis of the expression levels of microRNAs according to PCOS phenotype showed that within our study group women with oligo-amenorrhea had a significantly higher expression of miR-30a-3p compared to women with eumenorrea, suggesting the existence of a possible association with menstrual dysfunction (S3 and S4).

Carletti and colleagues showed that the loss of miR-21 in mice results in reduced ovulation rates [36], but in our study no differences were found in women with oligo-anovulation (S3). A possible explanation is that the authors analyzed the expression of miR-21 in the granulosa cells, instead of serum. MiR-21 exerts a broad spectrum of functions and is ubiquitously expressed. Therefore, the local ovary modulation might be cloaked by the systemic expression, thus minimizing the potential of miR-21 as a circulating biomarker for the phenotype under investigation.

Statistically significant inverse relationship between androstenedione concentrations and expression of miR-29a and miR155 and between 17-OHPg and miR-155 (Figure 1(A-C); S5) is reported. Ding et al. [37] showed that miR-29a-3p was downregulated in PCOS patients. In our experimental setting, we report a statistically significant inverse relationship between androstenedione concentrations and expression of miR-29a (Figure 1(B); S5). Merging those results, this datum might reveal the existence of a link between hyperandrogenemia and the activity of the miR-29 family either comparing PCOS women with healthy controls and within the PCOS phenotype itself.

Murri et al. [19] proved a direct association between the concentration of testosterone and levels of expression of miR-155 and that it is widely modulated between PCOS patients and healthy control with different BMI. In comparison, in our study we found an inverse relationship between this microRNA and the concentrations of two adrenal hormones, androstenedione and 17OHPg. (Figure 1(A,C); S5). The major difference between the two studies is the sample used: Murri et al. used whole blood sample, so the contribution of nucleate cells is crucial in their evaluations. This miRNA is expressed and functional in $\mathrm{B}$ and $\mathrm{T}$ cells, so the serum contribution is likely negligible in their quantifications.

It is also crucial to emphasize that, as a result of treatment for a period of four months with antiandrogen therapy, there was a mild increase in the expression of miR-27b and a highly significant increase in the expression of miR-155 (Table 1; S7). MiR-27b was over-expressed in whole blood of women with PCOS [19]. MiR-27 has been described as implicated in regulating cholesterol homeostasis and fatty acid metabolism [38]. Our findings showed an increase of miR-27b after therapy (Table 1; S7A). This could be linked to the reduction in circulating androgens and/or could be a side effect of an estrogen-progestinic therapy, such as weight gain and alterations in the lipid profile.

On the matter of metabolic parameters, DIo was positively associated with miR-103 and LDL cholesterol and inversely with miR-30a-3p and miR-320 (Figure $1(\mathrm{D}, \mathrm{F})$; S6). DIo provides a measure of beta cell function in relation to insulin-sensitivity. Over-expression of miR-103 may indicate adequate adaptation of beta cells to the condition of insulin resistance (as compensatory hyperinsulinemia) and therefore it seems to be protective for the development of type 2 diabetes. This is in contrast with the work of Trajkovski [25], who combined high levels of miR-103 in murine adipocytes with worsening of glucose homeostasis, but the possibility of a compensatory mechanism cannot be excluded. High levels of LDL cholesterol and low serum concentrations of HDL cholesterol are widely recognized to be major risk factors for cardiovascular disease $[39,40]$. Our data showed an inverse correlation between serum levels of miR-30a-3p and miR-320 and LDL cholesterol concentrations (Figure 1(E,F)).

In the light of the data obtained from our and previous studies, the most important role as a potential biomarker to monitor hyperandrogenemia in PCOS and the possible effect of an antiandrogen therapy can be attributed to miR-155. Our study both describes its inverse correlation with androstenedione and $17 \mathrm{OHPg}$ in hyperandrogenic PCOS patients and shows that antiandrogenic treatment determines an increase in its expression.

\section{Conclusions}

We suggest that miR-155 level in sera might be used as a noninvasive biomarker to monitor the response to estroprogestinic therapy in hyperandrogenic PCOS patients.

\section{Acknowledgements}

We want to thank all the collaborators and dedicate this paper to Dr. Marco Amato, who died of leukemia during the preparation of this paper. Without his expertise, without his medical knowledge of PCOS, and without his statistical skills this paper would have never been written.

\section{Disclosure statement}

No potential conflict of interest was reported by the authors.

\section{Funding}

This work was supported by FESR PON [2007/2013 4.1.1.1 RIMEDRI].

\section{ORCID}

Walter Arancio (D) http://orcid.org/0000-0002-6736-7734

\section{References}

1. Yildiz BO, Bozdag G, Yapici Z, et al. Prevalence, phenotype and cardiometabolic risk of polycystic ovary syndrome under different diagnostic criteria. Hum Reprod 2012;27:3067-73. 
2. Legro RS, Arslanian SA, Ehrmann DA, et al. Diagnosis and treatment of polycystic ovary syndrome: an endocrine society clinical practice guideline. J Clin Endocr Metab 2013;98:4565-92.

3. Galluzzo A, Amato MC, Giordano C. Insulin resistance and polycystic ovary syndrome. Nutr Metab Cardiovasc Dis 2008;18:511-8.

4. Amato MC, Vesco R, Vigneri E, et al. Hyperinsulinism and polycystic ovary syndrome (PCOS): role of insulin clearance. J Endocrinol Invest 2015;38:1319-26.

5. Amato MC, Magistro A, Gambino G, et al. Visceral adiposity index and DHEAS are useful markers of diabetes risk in women with polycystic ovary syndrome. Eur J Endocrinol 2015;172:79-88.

6. Amato MC, Giordano C, Galia M, AlkaMeSy Study Group, et al. Visceral adiposity index: a reliable indicator of visceral fat function associated with cardiometabolic risk. Diabetes Care 2010;33:920-2.

7. Lindholm A, Andersson L, Eliasson M, et al. Prevalence of symptoms associated with polycystic ovary syndrome. Int J Gynaecol Obstet 2008;102:39-43.

8. Xu N, Azziz R, Goodarzi M. Epigenetics in polycystic ovary syndrome: a pilot study of global dna methylation. Fertil Steril 2010; 94:781-3.e1.

9. Welt CK, Carmina E. Life cycle of polycystic ovary syndrome(PCOS): from in utero to menopause. J Clin Endocrinol Metab 2013;98: 4629-38.

10. Eriksen MB, Nielsen MFB, Brusgaard $K$, et al. Genetic alterations within the DENND1A gene in patients with polycystic ovary syndrome (PCOS). PLoS One 2013;8:e77186.

11. Ilie IR, Georgescu CE. Polycystic ovary syndrome-epigenetic mechanisms and aberrant microRNA. Adv Clin Chem 2015;71:25-45.

12. Bartel D. MicroRNAs: genomics, biogenesis, mechanism, and function Cell 2004;116:281-97.

13. Esquela-Kerscher A, Slack FJ. Oncomirs - microRNAs with a role in cancer. Nat Rev Cancer 2006;6:259-69.

14. Osmai M, Osmai $\mathrm{Y}$, Bang-Berthelsen $\mathrm{CH}$, et al. MicroRNAs as regulators of beta-cell function and dysfunction. Diabetes Metab Res Rev 2016;32:334-49.

15. Xu J, Zhao J, Evan G, et al. Circulating microRNAs: novel biomarkers for cardiovascular diseases. J Mol Med (Berl) 2012;90:865-75.

16. Heneghan HM, Miller N, Kerin MJ. Role of microRNAs in obesity and the metabolic syndrome. Obes Rev 2010;11:354-61.

17. Wei S, Xu H, Kuang Y. Systematic enrichment analysis of microRNA expression profiling studies in endometriosis. Iran J Basic Med Sci 2015;18:423-9.

18. Ranjha R, Paul J. Micro-RNAs in inflammatory diseases and as a link between inflammation and cancer. Inflamm Res 2013;62:343-55.

19. Murri M, Insenser M, Fernández-Durán E, San-Millán JL, EscobarMorreale HF, Effects of polycystic ovary syndrome (PCOS), sex hormones, and obesity on circulating miRNA-21, miRNA-27b, miRNA-103, and miRNA-155 expression. J Clin Endocr Metab 2013;98:E1835-44.

20. Long W, Zhao C, Ji C, et al. Characterization of serum microRNAs profile of PCOS and identification of novel non-invasive biomarkers. Cell Physiol Biochem 2014;33:1304-15.

21. Zhang H, Jiang X, Zhang Y, et al. MicroRNA 376a regulates follicle assembly by targeting pcna in fetal and neonatal mouse ovaries. Reproduction 2014;148:43-54
22. Sirotkin AV, Laukov M, Ovcharenko D, et al. Identification of microRNAs controlling human ovarian cell proliferation and apoptosis. J Cell Physiol 2010;223:49-56.

23. Roth LW, McCallie B, Alvero R, et al. Altered microRNA and gene expression in the follicular fluid of women with polycystic ovary syndrome. J Assist Reprod Genet 2014;31:355-62.

24. McCallie B, Schoolcraft WB, Katz-Jaffe MG. Aberration of blastocyst microRNA expression is associated with human infertility. Fertil Steril 2010;93:2374-82.

25. Trajkovski M, Hausser J, Soutschek J, et al. MicroRNAs 103 and 107 regulate insulin sensitivity. Nature 2011;474:649-53.

26. ChenY H, Heneidi S, Lee J-M, et al. MiRNA-93 inhibits GLUT4 and is overexpressed in adipose tissue of polycystic ovary syndrome patients and women with insulin resistance. Diabetes 2013;62:2278-86.

27. Sørensen AE, Wissing ML, Salö S, et al. MicroRNAs related to polycystic ovary syndrome (PCOS). Genes (Basel) 2014;5:684-708.

28. Rotterdam ESHRE/ASRM-Sponsored PCOS Consensus Workshop Group. Revised 2003 consensus on diagnostic criteria and long-term health risks related to polycystic ovary syndrome. Fertil Steril 2004;81:19-25

29. Amato MC, Verghi M, Galluzzo A, Giordano C. The oligomenorrhoic phenotypes of polycystic ovary syndrome are characterized by a high visceral adiposity index: a likely condition of cardiometabolic risk. Hum Reprod 2011;26:1486-94.

30. Ferriman D, Gallwey JD. Clinical assessment of body hair growth in women. J Clin Endocr Metab 1961;21:1440-7.

31. Utzschneider KM, Prigeon RL, Faulenbach MV, et al. Oral disposition index predicts the development of future diabetes above and beyond fasting and 2-h glucose levels. Diabetes Care 2009;32:335-41.

32. Matsuda $M$, DeFronzo RA. Insulin sensitivity indices obtained from oral glucose tolerance testing: comparison with the euglycemic insulin clamp. Diabetes Care 1999;22:1462-70.

33. DeFronzo RA, Matsuda M. Reduced time points to calculate the composite index. Diabetes Care 2010;33:e93.

34. Pfaffl MW. A new mathematical model for relative quantification in real-time RT-PCR. Nucleic Acids Res 2001;29:e45.

35. Diamanti-Kandarakis E, Mitrakou A, Hennes MM, et al. Insulin sensitivity and antiandrogenic therapy in women with polycystic ovary syndrome. Metabolism 1995;44:525-31.

36. Carletti MZ, Fiedler SD, Christenson LK. MicroRNA 21 blocks apoptosis in mouse periovulatory granulosa cells. Biol Reprod 2010;83: 286-95.

37. Ding C-F, Chen W-Q, ZhuY T, et al. Circulating microRNAs in patients with polycystic ovary syndrome. Hum Fertil 2015;18:22-9.

38. Fernández-Hernando C, Suárez Y, Rayner KJ, Moore KJ, MicroRNAs in lipid metabolism. Curr Opin Lipidol 2011;22:86-92.

39. Aranda JF, Madrigal-Matute J, Rotllan N, Fernández-Hernando C. MicroRNA modulation of lipid metabolism and oxidative stress in cardiometabolic diseases. Free Radical Bio Med 2013;64:31-9.

40. Assmann G, Schulte H. Relation of high-density lipoprotein cholesterol and triglycerides to incidence of atherosclerotic coronary artery disease (the PROCAM experience). Prospective cardiovascular Münster study. Am J Cardiol 1992;70:733-7. 